\title{
Stability of the solution of stochastic partial differential equation with random parameters
}

\author{
I. V. Yurchenko, V. S. Sikora \\ https://doi.org/10.31174/NT2018-158VI18-05 \\ Yuriy Fedkovych Chernivtsi National University, Chernivtsi, Ukraine \\ Corresponding author. E-mail:i.yurchenko@chnu.edu.ua
}

Paper received 24.01.18; Accepted for publication 30.01.18.

\begin{abstract}
The existence of a strong solution of the linear stochastic partial differential equation (LSPDE) in the corresponding space with random parameters is proved. The sufficient conditions are obtained for the asymptotic stability and mean square instability of the strong solution of the LSPDE.

Keywords: stochastic partial differential equation, mean square stability, asymptotic stability.
\end{abstract}

Introduction. Deterministic partial differential equations were considered by many authors, see, for example, [1-3] and bibliography therein.

Since the concepts of stochastic differential and integral and change of variables for a stochastic differential have been introduced and a strong solution to a stochastic differential equation (SDE) has been defined in the wellknown monographs [4-6] and then propagated to classes of stochastic functional differential equations [7-9] (see the extensive bibliography in these studies), it became possible to investigate an asymptotically strong solution for SPDE (see, for example, [5, 10-12], etc.).

The further analysis of SPDE involves the construction of mathematical models of complicated real systems, which need random parameters to be considered in these equations (see $[6,7,12,13]$, etc.).

In the paper, we will analyze the asymptotic behavior of strong solution of LSPDE taking into account random parameters in the left-hand side $[10,12]$.

Problem statement. Consider a stochastic experiment with the basic probability space $[1,4,5,7](\Omega, \mathcal{F}, \mathrm{F}, \mathrm{P})$, $\mathrm{F} \equiv\left\{\mathcal{F}_{t}, t \geq 0\right\} \quad$ is filtration, where function $u(t, x, \omega) \in \mathbb{R}^{1}$ is given, which is measurable with probability one in $t$ and $x$ with respect to the minimum $\sigma$ algebra $\mathcal{B}\left([0, T], \mathbb{R}^{1}\right)$ of Borel sets on the plane [13] and for which

$$
\int_{-\infty}^{+\infty} \mathrm{E}\left\{|u(t, x, \omega)|^{2}\right\} d x<\infty
$$

for all $t \in[0, T], E\{\cdot\}$ is expectation [14], and $T \subset[0, \infty)$. Denote by $M_{T}$ the space of function $\{u(t, x, \omega)\}$, which possesses the integrability property (1).

Introduce the norms $[6,15]$ :

$$
\begin{gathered}
\|u(t, x, \omega)\|_{L_{2 \mathrm{R}^{1}}}^{2} \equiv \int_{-\infty}^{+\infty}|u(t, x, \omega)|^{2} d x ; \\
\|\left. u(t, x, \omega)\right|_{L_{2 T}} ^{2} \equiv \int_{0}^{T}|u(t, x, \omega)|^{2} d t ; \\
\mathrm{E}_{u}(t) \equiv \mathrm{E}\left\{\|u(t, x, \omega)\|_{\left.L_{2 R^{1}}^{2}\right\}}\right\}
\end{gathered}
$$

where $L_{2 \mathrm{R}^{1}}$ and $L_{2 T}$ are spaces of functions $\{u(t, x, \omega)\}$, which have the corresponding norms (2) and (3).

In space $M_{T}$, it is necessary to introduce the norm

$$
\|u(t, x, \omega)\|^{2} \equiv \int_{0}^{T} \mathrm{E}_{u}(t) d t=\int_{0}^{T} \mathrm{E}\left[\int_{-\infty}^{+\infty}|u(t, x, \omega)|^{2} d x\right]^{2} d t,(5)
$$

Denote

$$
Q(A(\xi(\omega)), q, p) \equiv \sum_{k=1}^{n} \sum_{j=1}^{m} a_{k j}(\xi(\omega)) q^{k} p^{j},(6)
$$

where $A \equiv\left\{a_{k j}\right\}$ is a real matrix composed of elements $a_{k j} \in \mathbb{R}^{1}$.

In space $\boldsymbol{M}_{T}$, consider a subspace $\boldsymbol{M}_{1 T} \subset \mathbf{M}_{T}$, for whose elements the inclusion

$$
Q\left(A, \frac{\partial}{\partial t}, \frac{\partial}{\partial x}\right) u(t, x, \omega) \in M_{T} \text { (7) }
$$

takes place.

On $(\Omega, \mathcal{F}, \mathrm{F}, \mathrm{P})$ consider the Cauchy problem for the linear stochastic partial differential equation (LSPDE)

$$
\begin{gathered}
\frac{\partial}{\partial t}\left[Q\left(A\left(\xi_{1}(\omega)\right), \frac{\partial}{\partial t}, \frac{\partial}{\partial x}\right) u(t, x, \omega)\right]+ \\
+Q\left(B\left(\xi_{2}(\omega)\right), \frac{\partial}{\partial t}, \frac{\partial}{\partial x}\right) u(t, x, \omega)= \\
=Q\left(C\left(\xi_{3}(\omega)\right), \frac{\partial}{\partial t}, \frac{\partial}{\partial x}\right) u(t, x, \omega) \frac{d w(t, \omega)}{d t},(8) \\
\left.Q\left(A\left(\xi_{1}(\omega)\right), \frac{\partial}{\partial t}, \frac{\partial}{\partial x}\right) u(t, x, \omega)\right|_{t=0}=[Q u]_{0},(9)
\end{gathered}
$$

where $Q$ is defined by (6), matrices $B \equiv\left\{b_{i j}\left(\xi_{2}(\omega)\right)\right\}_{i, j=1}^{k, n}, \quad b_{i j}\left(\xi_{2}(\omega)\right) \in \mathbb{R}^{1}$; $C \equiv\left\{c_{i j}\left(\xi_{3}(\omega)\right)\right\}_{i, j=1}^{k, n}, \quad c_{i j}\left(\xi_{3}(\omega)\right) \in \mathbb{R}^{1}, \quad$ where $\xi_{i}(\omega), i=1,2,3$, are random value specified by the density $p_{\xi_{i}}(x), i=1,2,3$, (or by the distribution function $\left.F_{\xi_{i}}(x) \equiv \mathrm{P}\left\{\omega: \xi_{i}(\omega)<x \forall x \in \mathrm{R}^{1}\right\}, i=1,2,3 \quad[14]\right), \quad w(t, \omega)$ is a one-dimensional Wiener process [11], and $\xi_{i}(\omega)$, $i=1,2,3$, does not depend on $w(t, \omega)$. 
By a strong solution of the Cauchy problem (8), (9) we will understand function $u(t, x, \omega)$ continuous in $t \in[0, T]$ with probability one, consistent with filtration $\left\{\mathcal{F}_{t}, t \in[0, T]\right\}$, and such that with probability one for each pair $(t, x)$ it satisfies the integral stochastic equation $[1,4,11]$

$$
\begin{gathered}
Q\left(A\left(\xi_{1}(\omega)\right), \frac{\partial}{\partial t}, \frac{\partial}{\partial x}\right) u(t, x, \omega)=[Q u]_{0}+ \\
+\int_{0}^{t} Q\left(B\left(\xi_{2}(\omega)\right), \frac{\partial}{\partial x}\right) u(s, x, \omega) d s+ \\
+\int_{0}^{t} Q\left(C\left(\xi_{3}(\omega)\right), \frac{\partial}{\partial s}, \frac{\partial}{\partial x}\right) u(s, x, \omega) d w(s, \omega), 10
\end{gathered}
$$

with the non-random initial conditions (9).

Existence of the solution of the Cauchy problem for LSPDE (8), (9) in space $M_{1 T}$

To establish the existence of a strong solution of the Cauchy problem for (8), (9), we will first prove an auxiliary result.

Lemma 1 [20]. The Fourier transform in $x$ [1] for function $u(t, x, \omega)$

$$
v(t, \sigma, \omega) \equiv \frac{1}{\sqrt{2 \pi}} \int_{-\infty}^{+\infty} e^{-i \sigma x} u(t, x, \omega) d x
$$

does not bring it out of the space $M_{T}$ for any finite $T \subset \mathbb{R}^{1}$.

Theorem 1 [20]. Let the following conditions be satisfied for the Cauchy problem (8), (9):

i.) the roots of polynomial

$$
P(\lambda(x), i \sigma) \equiv \lambda Q(A(x), \lambda, i \sigma)+Q(B(x), \lambda, i \sigma)
$$

for an arbitrary $x \in \mathbb{R}^{1}$ and $\sigma \neq 0$ satisfy the inequality $\operatorname{Re} \lambda(x) \leq \psi(\sigma)<0, \psi(0)=0$;

ii.) $\forall t \in[0, T]$ i $C(x) \equiv 0_{k \times n}$ the deterministic equation

$$
\begin{gathered}
\frac{\partial}{\partial t}\left[Q\left(A(x), \frac{\partial}{\partial t}, \frac{\partial}{\partial x}\right) u(t, x)\right]+ \\
+Q\left(B(x), \frac{\partial}{\partial t}, \frac{\partial}{\partial x}\right) \mathfrak{u}(t, x)=0
\end{gathered}
$$

has the solution $u(t, x)$ of the Cauchy problem in $L_{2 \mathrm{R}^{1}}$ with the initial conditions

$$
Q\left(A(x), \frac{\partial}{\partial t}, \frac{\partial}{\partial x}\right) u(t, x)=[Q u]_{0} ;(13,
$$

iii.) random variable $\xi_{i}(\omega), i=1,2,3$, does not depend on $w(t, \omega)$.

Then the stochastic Cauchy problem (8), (9) for $C(x) \neq 0_{k \times n}$ has a solution in space $\mathbf{M}_{1 T}$.

\section{Asymptotic mean square behavior of the strong solu-} tion of the LSPDE.

Lemma 2. Let conditions of Theorem 1 be satisfied for the LSPDE (8), (9). Then:

i.) for an arbitrary matrix $C(x) \neq 0_{k \times n}$ the inclusion holds

$$
\mathrm{E}\left|Q\left(C\left(\xi_{3}(\omega)\right), d t, i \sigma\right)\right|^{2} H(t, \sigma) \in L_{2,(0,+\infty)}
$$

ii.) for the corresponding norm of this space, the equality is true

$$
\begin{gathered}
\mathbb{E}\left\|Q\left(C\left(\xi_{3}(\omega)\right), d t, i \sigma\right) H(t, \sigma)\right\|_{L_{2 T}}^{2}= \\
=\frac{1}{2 \pi} \int_{-\infty}^{+\infty} \frac{\mathbb{E}\left|Q\left(C\left(\xi_{3}(\omega)\right), i \lambda, i \sigma\right)\right|^{2}}{|P(i \lambda, i \sigma)|^{2}} d \lambda \equiv S(\sigma) .
\end{gathered}
$$

Proof. Using condition (i) of Theorem 1, we can calculate

$$
\begin{gathered}
\frac{1}{2 \pi} \int_{0}^{\infty}\left[Q\left(C\left(\xi_{3}(\omega)\right), d t, i \sigma\right) H(t, \sigma) e^{-i \lambda t}\right]= \\
=\frac{1}{2 \pi} \frac{Q\left(C\left(\xi_{3}(\omega)\right), i \lambda, i \sigma\right)}{P(i \lambda, i \sigma)}
\end{gathered}
$$

and multiplying the left- and right-hand sides of previous formula by $\mathbb{E}\left\{\mathbb{C}^{2}\right\}$ we obtain statement (14).

To prove (15), let us apply the Plancherel theorem [1]:

$$
\begin{gathered}
\mid Q\left(C\left(\xi_{3}(\omega)\right), d t, i \sigma\right) H(t, \sigma) \|_{L_{2(0, \sigma)}}^{2}= \\
=\frac{1}{2 \pi} \int_{-\infty}^{+\infty} \frac{\left|Q\left(C\left(\xi_{3}(\omega)\right), i \lambda, i \sigma\right)\right|^{2}}{|P(i \lambda, i \sigma)|^{2}} d \lambda \equiv S_{1}(\sigma) .
\end{gathered}
$$

Multiplying the left- and right-hand sides of the resultant equality by $E\left\{\left.|\cdot|\right|^{2}\right\}$, we get $S(\sigma)$ in formula (15).

Theorem 2. Let the conditions of Theorem 1 be satisfied. Then:

i.) if $\sup _{\sigma} S(\sigma)<1$, then $\lim _{t \rightarrow \infty} \mathrm{E}_{U}(t)=0$, where

$$
U(t, x, \omega) \equiv Q\left(D(\xi(\omega)), \frac{\partial}{\partial t}, \frac{\partial}{\partial x}\right) u(t, x, \omega)
$$

for an arbitrary real matrix $D$;

ii.) if $S(\sigma)>1$ on the set of the positive Lebesgue measure, then $\lim _{t \rightarrow \infty} \mathrm{E}_{U}(t)=+\infty$.

Proof. Since the positive kernel tends to zero as $t \rightarrow+\infty$, it follows [20] that $z(t, \sigma)$ tends to zero for $S(\sigma)<1, \sigma \neq 0$.

If the inequality $S(\sigma)<1$, in (14) holds, then it can be easily seen that as $t \rightarrow+\infty$, the absolute value of the Fourier transform $U(t, x, \omega)$ tends to zero for an arbitrary real matrix [19], uniformly with respect to $\sigma$ if $\sup _{\sigma} S(\sigma)<1$. It remains to pass to the limit under the sign of the Lebesgue integral to prove the first part of Theorem 2.

To prove the second part of Theorem 2, it will suffice to prove that $\lim _{t \rightarrow \infty} \int_{-\infty}^{+\infty} z(t, \sigma) d \sigma=\infty$, since (14) holds.

Indeed, let $S(\sigma)>1$ on the set $\Lambda$ of the positive Lebesgue measure, then $\lim _{t \rightarrow+\infty} z(t, \sigma)=+\infty$, since $z(t, \sigma)>0$. Theorem 2 is proved.

Problem of the loss of stability of a rod. In [3], the 
behavior of a rod subject to "white noise" is analyzed. Let the mathematical model of this process be the following stochastic partial differential equation with the derivative of the Wiener process that does not exist with probability one and is called "white noise," namely:

$$
\begin{gathered}
\frac{\partial^{4} u}{\partial x^{4}}-a\left(\xi_{1}(\omega)\right) \frac{\partial^{2} u}{\partial x^{2}}+b\left(\xi_{2}(\omega)\right) \frac{\partial^{2} u}{\partial t^{2}}- \\
-c\left(\xi_{3}(\omega)\right) \frac{\partial u}{\partial t}=\frac{\partial^{2} u}{\partial x^{2}} \frac{d w(t, \omega)}{d t},(16)
\end{gathered}
$$

with the initial conditions

$$
u(0, x)=f_{1}(x), \frac{\partial u(0, x)}{\partial t}=f_{2}(x)(17)
$$

and boundary conditions

$$
\begin{gathered}
u(t, 0)=u(t, l)=\frac{\partial u(t, 0)}{\partial x}=\frac{\partial u(t, l)}{\partial x}= \\
=\frac{\partial^{2} u(t, 0)}{\partial x^{2}}=\frac{\partial^{2} u(t, l)}{\partial x^{2}}=0 . \text { (18) }
\end{gathered}
$$

Here $\quad a\left(\xi_{1}(\omega)\right)>0, b\left(\xi_{2}(\omega)\right)>0, c\left(\xi_{3}(\omega)\right)>0$ with probability 1 . Similarly to the discrete case [3], the statistical stability margin $S_{a}^{2}$ with respect to the parameter $a(x), \forall x \in \mathbb{R}^{1}$, is determined as the most admissible intensity of processes with mutually independent values for which the system is stable in l.i.m., i.e., the solution is stabilized to zero.

As a result, we can calculate the statistical stability margin [17] $S_{k_{1} k_{2}}$ of system (16)-(18)

$$
S_{k_{1} k_{2}} \equiv \sum_{k=0}^{m} a_{k_{1} k_{2}}(\xi(\omega)) \frac{\partial^{k} u(t, x)}{\partial t^{k_{1}} \partial x^{k_{2}}}
$$

with respect to parameters $a_{k_{1} k_{2}}\left(\xi_{1}(\omega)\right), k=k_{1}+k_{2}$. If we denote

$$
P(\lambda, \sigma, \omega) \equiv \sum_{k=0}^{m} a_{k_{1} k_{2}}\left(\xi_{1}(\omega)\right) \lambda^{k_{1}}(i \sigma)^{k_{2}},
$$

then the statistical stability margin $S_{k_{1} k_{2}}(x)$ of the system can be calculated by the formula

$$
S_{k_{1} k_{2}}(x) \equiv\left[\sup _{\sigma} \frac{1}{2 \pi} \int_{-\infty}^{+\infty} \frac{|\lambda|^{k_{1}}|\sigma|^{k_{2}}}{|P(i \lambda, \sigma, x)|} d \lambda\right]^{-1} .
$$

Using the above statement (20), the statistical stability margin $S(x)$ with respect to the parameters $a(x), b(x), c(x)$ of system (16)-(18) is found:

$$
\begin{gathered}
S(x) \equiv \\
\equiv\left[\sup _{\sigma} \frac{1}{2 \pi} \int_{-\infty}^{+\infty} \frac{\sigma^{2} d \lambda}{\left(\sigma^{4}+a(x) \sigma^{2}-b(x) \lambda^{2}\right)^{2}+c(x)^{2} \lambda^{2}}\right]^{-1}= \\
=2 a(x) c(x), \forall x \in \mathbb{R}^{1} .(21)
\end{gathered}
$$

Thus, system (16)-(18) is stable in 1.i.m., for which $S(x)>\varepsilon^{2}, \forall x \in \mathrm{R}^{1}$.

Let the right-hand side of Eq. (16) in system (16)-(18) be subject to external random disturbances $\xi(\omega)$. This becomes possible if we place the system on a platform whose inching movement can be described by $\varphi(\xi(\omega))$. Then (16) becomes

$$
\begin{aligned}
& \frac{\partial^{4} u}{\partial x^{4}}-a \frac{\partial^{2} u}{\partial x^{2}}+b \frac{\partial^{2} u}{\partial t^{2}}-c \frac{\partial u}{\partial t}= \\
& =\varphi(\xi(\omega)) \frac{\partial^{2} u}{\partial x^{2}} \frac{d w(t, \omega)}{d t} .(22)
\end{aligned}
$$

Using the definition of the statistical stability margin for system (22), (17), (18), we get

$$
\begin{gathered}
\equiv\left[\mathrm{E}\left\{|\varphi(\xi)|^{2}\right\} \sup _{\sigma} \frac{1}{2 \pi} \int_{-\infty}^{+\infty} \frac{\sigma^{2} d \lambda}{\left(\sigma^{4}+a \sigma^{2}-b \lambda^{2}\right)^{2}+c^{2} \lambda^{2}}\right]^{-1}= \\
=\mathrm{E}\left\{|\varphi(\xi)|^{2}\right\} 2 a c . \text { (23) }
\end{gathered}
$$

Applying the sufficient conditions of stability in l.i.m. from Theorem 2, we conclude that system (22), (17), (18) is stable in 1.i.m. if $E\left\{\varphi^{2}(\xi)\right\} 2 a c<1$, and is unstable in 1.i.m. otherwise.

Let $\xi(\omega)$ have the distribution law

$$
P\{\omega: \xi \equiv \mathbf{1}\}=P\{\omega: \xi=-1\}=\frac{1}{2}
$$

and $\varphi(\xi(\omega)) \equiv \xi(\omega)$. Then $\mathrm{E}\{\xi\}=0, D\{\xi\}=1$ and condition (23) coincides with condition (21).

If for the distribution law of $\xi(\omega)$ we take the Poisson law

$$
\mathrm{P}\{\omega: \xi=k\}=\frac{\lambda^{k}}{k !} e^{-\lambda}
$$

and $\varphi(\xi)=\xi$, тоді $\mathrm{E} \xi=D \xi=\lambda$. Therefore, the condition of stability in 1.i.m. of system (22), (17), (18) becomes $2 a c \lambda<1$, and that of instability, respectively, $2 a c \lambda>1$.

Conclusions. The stochastic model of complicated systems proposed in the paper is apparently the first attempt to take randomness into account to the fullest extent in the analysis of real processes described by partial differential equations whose right-hand sides consider not only diffusion disturbances such as Brownian process [5, 10, 18, 19] but random disturbances of other types as well.

\section{REFERENCES}

[1] R. Bellman and K.L. Cooke, Differential-Difference Equa- [4] A.V. Gulinskii and A.N. Shiryaev, Theory of Random Protions, Academic Press, New York (1963).

[2] S.G. Mikhlin, Linear Partial Differential Equations [in Russian], Nauka, Moscow (1997).

[3] V.V. Stepanov, A Course in Differential Equations [in Russian], Nauka, Moscow (1978). cesses [in Russian], Fizmatlit, Moscow (2005).

[5] I.I. Gikhman and A.V. Skorokhod, Stochastic Differential Equations and their Application [in Russian], Naukova Dumka, Kyiv (1980).

[6] I.I. Gikhman and A.V. Skorokhod, Controlled Random Pro- 
cesses [in Russian], Naukova Dumka, Kyiv (1977).

[7] V.S. Koroliuk, E.F. Tsarkov, and V.K. Yasynskyy, Probability, Statistics, and Random Processes. Theory and Computer Practice, in 3 vols., Vol. 3. Random Processes [in Ukrainian], Zoloti Lytavry, Chernivtsi (2009).

[8] E.F. Tsarkov and V.K. Yasynskyy, Quasilinear Stochastic Functional Differential Equations [in Russian], Orientir, Riga (1992).

[9] E.F. Tsarkov, Random Disturbances of Functional Differential Equations under Random Disturbances of their Parameters [in Russian], Zinatne, Riga (1989).

[10] I.I. Gikhman and A.V. Skorokhod, Stochastic Partial Differential Equations. A Collection of Sci. Papers [in Russian], Inst. Matem. AN UkrSSR, Kyiv (1981), pp. 25-59.

[11] A.Ya. Dorogovtsev, S.D. Ivasishen, and A.G. Kukush, "Asymptotic behavior of solutions of the heat-conduction equation with white noise in the right side," Ukr. Math. J., 37, No. 1, 10-15 (1985).

[12] G.M. Perun and V.K. Yassinskii, "Investigation of the Cauchy problem for stochastic partial differential equations," Ukr. Mat. Zhurn., 45, No. 9, 1413-1420 (1993).

[13] E.B. Dynkin, Markov Processes [in Russian], Fizmatgiz, Moscow (1969).

[14] V.S. Koroliuk, E.F. Tsarkov, and V.K. Yasynskyy, Probability, Statistics, and Random Processes. Theory and Computer Practice, in 3 vols., Vol. 1. Probability [in Ukrainian],
Zoloti Lytavry, Chernivtsi (2007).

[15] A.N. Kolmogorov and S.V. Fomin, Elements of the Theory of Functions and Functional Analysis [in Russian], Nauka, Moscow (1976).

[16] R.Z. Khas'minskii, Stability of Systems of Differential Equations under Random Disturbances of their Parameters [in Russian], Nauka, Moscow (1969).

[17] S.D. Eidelman, Parabolic Systems [in Russian], Nauka, Moscow (1964).

[18] N.P. Donez, I.V. Yurchenko, V.K. Yasynskyy, "Mean Square Behavior of the Strong Solution of a Linear nonAutonomous Stochastic Partial Differential Equation with Markov Parameters", Cybernetics and System Analysis, 50, No. 6, 930-939 (2014). doi:10.1007/s10559-014-9683-8

[19] V.S. Koroliuk, I.V. Yurchenko, V.K. Yasynskyy, "Behavior of the Second Moment of the Solution to the Autonomous Stochastic Linear Partial Differential Equation with Random Parameters in the Right-Hand Side", Cybernetics and Systems Analysis, 51, No. 1, 56-63(2015).doi:10.1007/s10559. 015-9697-x.

[20] I.V. Yurchenko, V.K. Yasynskyy, "On stability of the strong solution of the autonomous stochastic partial differential equation with random parameters", Sworld Journal, Issue №12 (Scientific world, Ivanovo, 2017), 343-356. DOI: 10.21893/2227-6920.2017-12.012.

Устойчивость решения стохастического дифференциального уравнения в частных производных со случайными параметрами

\section{И. В. Юрченко, В. С. Сикора}

Аннотация. Доказано существование сильного решения линейного стохастического дифференциального уравнения в частных производных в соответствующем пространстве со случайными параметрами. Получены достаточные условия для асимптотической устойчивости и среднеквадратичной неустойчивости сильного решения.

Ключевые слова: стохастические дифференциальные уравнения в частных производных, устойчивость в среднем квадратическом, асимптотическая устойчивость. 